\title{
Global variational solutions to a class of fractional spde's on unbounded domains
}

\author{
Marco Dozzi*, Rim Touibi*, Pierre- A. Vuillermot*,** \\ UMR-CNRS 7502, Inst. Élie Cartan de Lorraine, Nancy, France*, \\ Grupo de Física Matemática, GFMUL, Faculdade de Ciências, \\ Universidade de Lisboa, 1749-016 Lisboa, Portugal**
}

\begin{abstract}
In this article we prove new results regarding the existence and the uniqueness of global variational solutions to Neumann initial-boundary value problems for a class of non-autonomous stochastic parabolic partial differential equations. The equations we consider are defined on unbounded open domains in Euclidean space satisfying certain geometric conditions, and are driven by a multiplicative noise derived from an infinite-dimensional fractional Wiener process characterized by a sequence of Hurst parameters $\mathbf{H}=\left(\mathrm{H}_{i}\right)_{i \in \mathbb{N}^{+}} \subset\left(\frac{1}{2}, 1\right)$. These parameters are in fact subject to further constraints that are intimately tied up with the nature of the nonlinearity in the stochastic term of the equations, and with the choice of the functional spaces in which the problem at hand is well-posed. Our method of proof rests on compactness arguments in an essential way.
\end{abstract}

\section{Outline and statement of the main result}

Many applications ranging from the worlds of engineering and finance to the natural sciences call for a mathematical modelling in terms of stochastic differential equations. In particular, there have been many works devoted to the analysis of the ultimate behavior of solutions to stochastic partial differential equations of parabolic type which specifically occur in population dynamics, population genetics, nerve pulse propagation and related topics, to name only a few (see, e.g., [19] for a brief account of some of those works and the references therein). Moreover, there have also been several more recent articles dealing with the analysis of solutions to various types of semilinear parabolic stochastic partial differential equations driven either by a Brownian noise, or by a fractional noise with Hurst parameter $\mathrm{H} \in\left(\frac{1}{2}, 1\right)$ (see, e.g., [3], [4, [5- [7, and the plethora of references therein, particularly [11]). While these works have been primarily centered around questions of global existence, uniqueness and blowup in finite time, there have also been investigations essentially motivated by issues in financial mathematics devoted to the analysis of problems that involve 
a mixture of a Brownian noise with a fractional noise, within the realm of both ordinary and partial stochastic differential equations (see, e.g., [9, [13-15] and the references therein).

It is our purpose here to contribute further to the analysis of some of the above questions by proving the existence, and in some cases the uniqueness, of global variational solutions to Neumann initial-boundary value problems associated with a class of non-autonomous stochastic parabolic partial differential equations defined on certain regions of Euclidean space. In what follows we assume that all the functional spaces are real, use the standard notations for the usual spaces of Lebesgue integrable functions and their norms, and begin by defining the Wiener process that will generate the fractional noise we need. Let $D \subset \mathbb{R}^{d}$ be an unbounded open domain where $d \in \mathbb{N}^{+}$, and let us consider a linear, self-adjoint, positive, non-degenerate trace-class operator $C$ in $L^{2}(D)$ whose eigenfunctions and eigenvalues we write $\left(e_{i}\right)_{i \in \mathbb{N}^{+}}$and $\left(\lambda_{i}\right)_{i \in \mathbb{N}^{+}}$, respectively. Let $\mathrm{H}=\left(\mathrm{H}_{i}\right)_{i \in \mathbb{N}^{+}}$be a sequence of Hurst parameters satisfying $\mathrm{H}_{i} \in\left(\frac{1}{2}, 1\right)$ for every $i$, and for $t \in \mathbb{R}_{0}^{+}$let $\left(B^{\mathrm{H}_{i}}(t)\right)_{i \in \mathbb{N}^{+}}$be a sequence of onedimensional, independent fractional Brownian motions defined on the complete probability space $(\Omega, \mathcal{F}, \mathbb{P})$ and starting at the origin. Assuming that $\left(e_{i}\right)_{i \in \mathbb{N}^{+}}$ constitutes an orthonormal basis of $L^{2}(D)$, we then define the $L^{2}(D)$-valued fractional Wiener process $W^{\mathrm{H}}(., t)$ by

$$
W^{\mathrm{H}}(., t):=\sum_{i=1}^{+\infty} \sqrt{\lambda_{i}} e_{i}(.) B^{\mathrm{H}_{i}}(t) .
$$

This series converges strongly in $L^{2}(D) \mathbb{P}$-a.s. by virtue of the basic properties of $B^{\mathrm{H}_{i}}(t)$, the boundedness of the sequence $\mathrm{H}$ and the fact that $C$ is traceclass. From this we conclude that (1) defines a centered Gaussian process whose covariance is entirely determined by $C$, that is,

$$
\begin{aligned}
& \mathbb{E}\left(\left(W^{\mathrm{H}}(., s), v\right)_{2}\left(W^{\mathrm{H}}(., t), \hat{v}\right)_{2}\right) \\
= & \frac{1}{2} \sum_{i=1}^{+\infty}\left(s^{2 \mathrm{H}_{i}}+t^{2 \mathrm{H}_{i}}-|s-t|^{2 \mathrm{H}_{i}}\right)\left(C v, e_{i}\right)_{2}\left(\hat{v}, e_{i}\right)_{2}
\end{aligned}
$$

for all $s, t \in \mathbb{R}_{0}^{+}$and all $v, \hat{v} \in L^{2}(D)$, where $(., .)_{2}$ denotes the standard inner product in this space and $\mathbb{E}$ the expectation functional on $(\Omega, \mathcal{F}, \mathbb{P})$ (see, e.g., [16] for a discussion of various basic properties of fractional Brownian motion). For $T \in \mathbb{R}^{+}$arbitrary, we then consider the class of stochastic initial-boundary value problems formally given by

$$
\begin{aligned}
d u(x, t) & =\left(\operatorname{div}\left(k(x, t) \nabla_{x} u(x, t)\right)+g(u(x, t))\right) d t+h(u(x, t)) W^{\mathrm{H}}(x, d t) \\
(x, t) & \in D \times(0, T] \\
u(x, 0) & =\varphi(x), \quad x \in D \\
\frac{\partial u(x, t)}{\partial n(k)} & =0, \quad(x, t) \in \partial D \times(0, T]
\end{aligned}
$$


where $\partial D=\bar{D} \backslash D$ stands for the boundary of $D$ and the last line for the conormal derivative of $u$ relative to the matrix-valued function $k$. Moreover, $g$ and $h$ are real-valued while $\varphi$ is a random initial condition. Regarding these functions we will need the following hypotheses:

(K) The function $k: D \times(0, T] \mapsto \mathbb{R}^{d^{2}}$ is Lebesgue-mesurable and we have $k_{i, j}()=.k_{j, i}($.$) for all i, j \in\{1, \ldots, d\}$. Moreover, there exist constants $\underline{k}, \bar{k} \in \mathbb{R}^{+}$ such that the inequalities

$$
\underline{k}|y|^{2} \leq(k(x, t) y, y) \leq \bar{k}|y|^{2}
$$

hold uniformly in $(x, t) \in D \times(0, T]$ for all $y \in \mathbb{R}^{d}$, where $(.,$.$) and |$.$| denote$ the standard Euclidean inner product and the related norm in $\mathbb{R}^{d}$, respectively.

(L) The functions $g, h: \mathbb{R} \mapsto \mathbb{R}$ are Lipschitz continuous. Moreover, the derivative $h^{\prime}$ exists, is Hölder continuous with exponent $\gamma \in(0,1]$ and bounded. In addition we impose that

$$
\underline{\mathrm{H}} \in\left(\frac{1}{\gamma+1}, 1\right)
$$

where $\underline{\mathrm{H}}:=\inf _{i \in \mathbb{N}^{+}} \mathrm{H}_{i}$.

(I) The initial condition $\varphi$ is an $L^{2}(D)$-valued random variable.

Finally, whereas the above properties of the operator $C$ are sufficient to define $W^{\mathrm{H}}$, they are not quite strong enough to allow us to prove the result we are looking for. Recalling that $C$ is necessarily an integral transform whose generating kernel we denote by $\kappa$, we still impose the following spectral condition:

(C) We have

$$
x \mapsto \int_{D} d y|\kappa(x, y)|^{2} \in L^{\infty}(D)
$$

and

$$
\sum_{i=1}^{+\infty} \sqrt{\lambda_{i}}\left\|e_{i}\right\|_{\infty}<+\infty
$$

As to the consistency of this hypothesis, we simply remark that we have indeed $e_{i} \in L^{\infty}(D)$ as a consequence of (5), which follows easily from the eigenvalue equation $C e_{i}=\lambda_{i} e_{i}$, Schwarz inequality and the fact that $\left\|e_{i}\right\|_{2}=1$.

We will also need some functional spaces in order to define the notion of variational solution we are interested in. To this end we fix once and for all an $\alpha \in\left(1-\underline{\mathrm{H}}, \frac{\gamma}{\gamma+1}\right)$ where $\underline{\mathrm{H}}$ satisfies (44), and introduce the Banach space 
$\mathcal{B}^{\alpha, 2}\left([0, T] ; L^{2}(D)\right)$ of all Lebesgue measurable mappings $u:[0, T] \mapsto L^{2}(D)$ endowed with the norm

$$
\|u\|_{\alpha, 2, T}^{2}:=\left(\sup _{t \in[0, T]}\|u(t)\|_{2}\right)^{2}+\int_{0}^{T} d t\left(\int_{0}^{t} d \tau \frac{\|u(t)-u(\tau)\|_{2}}{(t-\tau)^{\alpha+1}}\right)^{2}<+\infty .
$$

Furthermore, let $H^{1}(D \times(0, T))$ be the isotropic Sobolev space consisting of all real-valued functions $v \in L^{2}(D \times(0, T))$ that possess distributional derivatives $v_{x_{i}}, v_{\tau} \in L^{2}(D \times(0, T))$, endowed with the inner product

$$
\begin{aligned}
\left(v_{1}, v_{2}\right)_{1,2, T}: & =\int_{D \times(0, T)} d x d \tau v_{1}(x, \tau) v_{2}(x, \tau) \\
& +\sum_{i=1}^{d} \int_{D \times(0, T)} d x d \tau v_{1, x_{i}}(x, \tau) v_{2, x_{i}}(x, \tau) \\
& +\int_{D \times(0, T)} d x d \tau v_{1, \tau}(x, \tau) v_{2, \tau}(x, \tau)
\end{aligned}
$$

and the corresponding norm

$$
\|v\|_{1,2, T}=(v, v)_{1,2, T}^{\frac{1}{2}} .
$$

We note that for any function $v \in H^{1}(D \times(0, T))$ which does not depend on time we have $v \in H^{1}(D)$, the usual Sobolev space on $D$ whose norm we denote by $\|\cdot\|_{1,2}$. Moreover, let $H^{1}((0, T))$ be the Sobolev space of functions defined on the time interval $(0, T)$. From these definitions it follows immediately that if $v \in H^{1}(D)$ and $\eta \in H^{1}((0, T))$ then $v \otimes \eta \in H^{1}(D \times(0, T))$. This allows us to consider the vector space of all finite linear combinations of such tensor products as an inner product space with respect to (8), and we write $\mathcal{H}(D \times(0, T))$ for its completion in $H^{1}(D \times(0, T))$ with respect to (9). About this Hilbert space we shall prove in Section 2 the existence of the trace embedding

$$
\mathcal{H}(D \times(0, T)) \rightarrow L^{2}(D \times\{\tau=t\})
$$

valid for each $t \in[0, T]$ and every $d \in \mathbb{N}^{+}$, which is important to formulate the following notion of variational solution:

Definition 1. We say the $H^{1}(D)$-valued random field $\left(u_{V, \varphi}(., t)\right)_{t \in[0, T]}$ defined on $(\Omega, \mathcal{F}, \mathbb{P})$ is a global variational solution to (2) if the following two conditions hold:

(1) We have $u_{V, \varphi} \in L^{2}\left(0, T ; H^{1}(D)\right) \cap \mathcal{B}^{\alpha, 2}\left([0, T] ; L^{2}(D)\right) \mathbb{P}$-a.s., which means that the relations

$$
\int_{0}^{T} d \tau\left\|u_{V, \varphi}(., \tau)\right\|_{1,2}^{2}=\int_{0}^{T} d \tau\left(\left\|u_{V, \varphi}(., \tau)\right\|_{2}^{2}+\left\|\nabla u_{V, \varphi}(., \tau)\right\|_{2}^{2}\right)<+\infty
$$

and

$$
\left\|u_{V, \varphi}\right\|_{\alpha, 2, T}<+\infty
$$


hold $\mathbb{P}$-a.s. In the sequel we shall sometimes write $\left\|u_{V, \varphi}\right\|_{L^{2}\left(0, T ; H^{1}(D)\right)}$ for norm (11).

(2) The integral relation

$$
\begin{aligned}
\left(v(., t), u_{V, \varphi}(., t)\right)_{2}= & (v(., 0), \varphi)_{2}+\int_{0}^{t} d \tau\left(v_{\tau}(., \tau), u_{V, \varphi}(., \tau)\right)_{2} \\
& -\sum_{i, j=1}^{d} \int_{0}^{t} d \tau\left(v_{x_{i}}(., \tau), k_{i, j}(., \tau) u_{V, \varphi, x_{j}}(., \tau)\right)_{2} \\
& +\int_{0}^{t} d \tau\left(v(., \tau), g\left(u_{V, \varphi}(., \tau)\right)\right)_{2} \\
& +\int_{0}^{t} \int_{D} d x v(x, \tau) h\left(u_{V, \varphi}(x, \tau)\right) W^{\mathrm{H}}(x, d \tau)
\end{aligned}
$$

holds $\mathbb{P}$-a.s. for each $v \in \mathcal{H}(D \times(0, T))$ and every $t \in[0, T]$, where $x \mapsto v(x, t) \in$ $L^{2}(D)$ stands for the trace of $v$ in the sense of (10), and where the stochastic integral with respect to (1) is defined as

$$
\begin{aligned}
& \int_{0}^{t} \int_{D} d x v(x, \tau) h\left(u_{V, \varphi}(x, \tau)\right) W^{\mathrm{H}}(x, d \tau) \\
:= & \sum_{i=1}^{+\infty} \sqrt{\lambda_{i}} \int_{0}^{t}\left(v(., \tau), h\left(u_{V, \varphi}(., \tau)\right) e_{i}\right)_{2} B^{\mathrm{H}_{i}}(d \tau) .
\end{aligned}
$$

In order to make sense out of each term in (12) and prove the existence of such a solution we will still need the following geometric hypothesis on the domain $D$ :

(D) There exists a sequence $\left(D_{N}\right)_{N \in \mathbb{N}^{+}}$of open sets such that for every $N$ we have $D_{N} \subset D_{N+1} \subset D$ and the compactness of the embedding

$$
H^{1}\left(D_{N}\right) \hookrightarrow L^{2}\left(D_{N}\right) .
$$

Moreover, let $\mathcal{B} \subset H^{1}(D)$ be any bounded set. Then for each $\epsilon>0$ there exists $N_{\epsilon} \in \mathbb{N}^{+}$such that

$$
\int_{D \backslash D_{N_{\epsilon}}} d x|u(x)|^{2}<\epsilon
$$

for every $u \in \mathcal{B}$.

REMARK. It is essential to observe here that any domain satisfying (D) is necessarily of finite Lebesgue measure as a consequence of the theory of tesselations developed in Chapter 6 of [1, and more importantly that (D) implies the compactness of the embedding $H^{1}(D) \hookrightarrow L^{2}(D)$ as it amounts to a geometric condition implying that $D$ becomes rapidly narrow at infinity, as the two examples discussed at the end of this section will show. It is also easily verified 
that each term in (12) is well defined and finite $\mathbb{P}$-a.s. as a consequence of all of the above hypotheses. In particular, we may conclude that (13) is an infinite sum of one-dimensional, pathwise, generalized Stieltjes integrals which defines a real-valued random variable as a consequence of Hypothesis $(\mathrm{C})$ and of the fact that $h$ is Lipschitz continuous. We shall dwell a bit more on this and on related properties of the stochastic integral in the Appendix. We note that Problem (2) was thoroughly analyzed in [17 in case $D$ is a bounded domain satisfying the cone condition and with a single Hurst parameter in (10).

Under these conditions our main result is the following:

Theorem. Assume that Hypotheses (K), (L), (I), (C) and (D) hold. Then Problem (2) possesses a global variational solution $u_{V, \varphi}$. Moreover, if $h$ is an affine function then $u_{V, \varphi}$ is the unique solution to (2).

In order to prove this result we shall organize the remaining part of this article in the following way: in Section 2 we first state the existence of $u_{V, \varphi}$ when the test functions in (12) are independent of time, that is, with $v \in H^{1}(D)$. We then extend the statement to the case of an approximating family of test functions in $\mathbb{R}^{d+1}$ consisting of finite linear combinations of the tensor products we alluded to above, and eventually to all test functions $v \in \mathcal{H}(D \times(0, T))$ by a suitable density argument and by invoking the trace embedding of the form (10) which we prove by elementary means. In Section 2 we also illustrate our results with two examples, while we prove some crucial estimates for the stochastic integral in the Appendix by means of a necessary modification of the theory set forth in [16] due to the fact that we are dealing there with an infinite sequence of Hurst parameters satisfying (4). The method rests in an essential way on a particular case of an inequality proved by Garsia, Rodemich and Rumsey in [8], and on Minkowski's integral inequality (see, e.g., Appendix A in [18]).

\section{Proof of the main result and two examples}

We begin with the following result:

Proposition 1. Assume that Hypotheses (K), (L), (I), (C) and (D) hold. Then there exists $u_{V, \varphi} \in L^{2}\left(0, T ; H^{1}(D)\right) \cap \mathcal{B}^{\alpha, 2}\left([0, T] ; L^{2}(D)\right)$ such that the integral relation (12) holds for every $v \in H^{1}(D)$, that is,

$$
\begin{aligned}
\left(v, u_{V, \varphi}(., t)\right)_{2}= & (v, \varphi)_{2}-\sum_{i, j=1}^{d} \int_{0}^{t} d \tau\left(v_{x_{i}}, k_{i, j}(., \tau) u_{V, \varphi, x_{j}}(., \tau)\right)_{2} \\
& +\int_{0}^{t} d \tau\left(v, g\left(u_{V, \varphi}(., \tau)\right)\right)_{2} \\
& +\int_{0}^{t} \int_{D} d x v(x) h\left(u_{V, \varphi}(x, \tau)\right) W^{\mathrm{H}}(x, d \tau)
\end{aligned}
$$


$\mathbb{P}$-a.s. for every $t \in[0, T]$.

Proof. Since the theory developed in Chapter 6 of 1 implies the compactness of the embedding $H^{1}(D) \hookrightarrow L^{2}(D)$, the result follows from a direct adaptation of all the arguments put forward in the first part of [17].

The next intermediary result is:

Lemma 1. Assume that Hypotheses $(K),(L),(I),(C)$ and $(D)$ hold and let $u_{V, \varphi}$ be the random field of Proposition 1. Then for all finite linear combinations $\hat{v}$ of functions of the form $v \otimes \eta$ where $v \in H^{1}(D)$ and $\eta \in H^{1}((0, T))$ we have

$$
\begin{aligned}
\left(\hat{v}(., t), u_{V, \varphi}(., t)\right)_{2}= & (\hat{v}(., 0), \varphi)_{2}+\int_{0}^{t} d \tau\left(\hat{v}_{\tau}(., \tau), u_{V, \varphi}(., \tau)\right)_{2} \\
& -\sum_{i, j=1}^{d} \int_{0}^{t} d \tau\left(\hat{v}_{x_{i}}(., \tau), k_{i, j}(., \tau) u_{V, \varphi, x_{j}}(., \tau)\right)_{2} \\
& +\int_{0}^{t} d \tau\left(\hat{v}(., \tau), g\left(u_{V, \varphi}(., \tau)\right)\right)_{2} \\
& +\int_{0}^{t} \int_{D} d x \hat{v}(x, \tau) h\left(u_{V, \varphi}(x, \tau)\right) W^{\mathrm{H}}(x, d \tau)
\end{aligned}
$$

$\mathbb{P}$-a.s. for every $t \in[0, T]$.

Proof. By linearity it is sufficient to prove that (17) holds for $\hat{v}=v \otimes \eta$. The easiest way out is to start with the first integral on the right-hand side. Using (16) we obtain

$$
\begin{aligned}
& \int_{0}^{t} d \tau\left(\hat{v}_{\tau}(., \tau), u_{V, \varphi}(., \tau)\right)_{2} \\
= & \int_{0}^{t} d \tau \eta^{\prime}(\tau)\left(v, u_{V, \varphi}(., \tau)\right)_{2} \\
= & (\hat{v}(., t), \varphi)_{2}-(\hat{v}(., 0), \varphi)_{2} \\
& -\sum_{i, j=1}^{d} \int_{0}^{t} d \tau \eta^{\prime}(\tau) \int_{0}^{\tau} d \sigma\left(v_{x_{i}}, k_{i, j}(., \sigma) u_{V, \varphi, x_{j}}(., \sigma)\right)_{2} \\
& +\int_{0}^{t} d \tau \eta^{\prime}(\tau) \int_{0}^{\tau} d \sigma\left(v, g\left(u_{V, \varphi}(., \sigma)\right)\right)_{2} \\
& +\int_{0}^{t} d \tau \eta^{\prime}(\tau) \int_{0}^{\tau} \int_{D} d x v(x) h\left(u_{V, \varphi}(x, \sigma)\right) W^{\mathrm{H}}(x, d \sigma) .
\end{aligned}
$$

We then integrate by parts the last three terms with respect to $\tau$ and reintroduce 
$\hat{v}$ whenever possible to obtain respectively

$$
\begin{aligned}
& \sum_{i, j=1}^{d} \int_{0}^{t} d \tau \eta^{\prime}(\tau) \int_{0}^{\tau} d \sigma\left(v_{x_{i}}, k_{i, j}(., \sigma) u_{V, \varphi, x_{j}}(., \sigma)\right)_{2} \\
= & \eta(t) \sum_{i, j=1}^{d} \int_{0}^{t} d \tau\left(v_{x_{i}}, k_{i, j}(., \tau) u_{V, \varphi, x_{j}}(., \tau)\right)_{2} \\
& -\sum_{i, j=1}^{d} \int_{0}^{t} d \tau\left(\hat{v}_{x_{i}}(., \tau), k_{i, j}(., \tau) u_{V, \varphi, x_{j}}(., \tau)\right)_{2}
\end{aligned}
$$

and

$$
\begin{aligned}
& \int_{0}^{t} d \tau \eta^{\prime}(\tau) \int_{0}^{\tau} d \sigma\left(v, g\left(u_{V, \varphi}(., \sigma)\right)\right)_{2} \\
= & \eta(t) \int_{0}^{t} d \tau\left(v, g\left(u_{V, \varphi}(., \tau)\right)\right)_{2}-\int_{0}^{t} d \tau\left(\hat{v}(., \tau), g\left(u_{V, \varphi}(., \tau)\right)\right)_{2}
\end{aligned}
$$

for the deterministic integrals, while we get

$$
\begin{aligned}
& \int_{0}^{t} d \tau \eta^{\prime}(\tau) \int_{0}^{\tau} \int_{D} d x v(x) h\left(u_{V, \varphi}(x, \sigma)\right) W^{\mathrm{H}}(x, d \sigma) \\
= & \eta(t) \int_{0}^{t} \int_{D} d x v(x) h\left(u_{V, \varphi}(x, \tau)\right) W^{\mathrm{H}}(x, d \tau) \\
& -\int_{0}^{t} \int_{D} d x \hat{v}(x, \tau) h\left(u_{V, \varphi}(x, \tau)\right) W^{\mathrm{H}}(x, d \tau)
\end{aligned}
$$

for the stochastic integral. The substitution of (19)-(21) into (18) then leads to the desired result, after having lumped together the three terms containing the factor $\eta(t)$ and used there (16) once again.

We infer from the preceding considerations that for any $v \in \mathcal{H}(D \times(0, T))$ there exist functions $\hat{v}_{n}$ satisfying (17) for every $n \in \mathbb{N}^{+}$such that

$$
\left\|v-\hat{v}_{n}\right\|_{1,2, T} \rightarrow 0
$$

as $n \rightarrow+\infty$, where $\|\cdot\|_{1,2, T}$ is given by (9). The first consequence of this is that we can already approximate the three deterministic integrals in (12), deferring to separate propositions the analysis of the remaining terms:

Proposition 2. Let $v \in \mathcal{H}(D \times(0, T))$ and let $\left(\hat{v}_{n}\right)_{n \in \mathbb{N}^{+}}$be as in (22). Then we have

$$
\lim _{n \rightarrow+\infty} \int_{0}^{t} d \tau\left(\hat{v}_{n, \tau}(., \tau), u_{V, \varphi}(., \tau)\right)_{2}=\int_{0}^{t} d \tau\left(v_{\tau}(., \tau), u_{V, \varphi}(., \tau)\right)_{2},
$$




$$
\begin{aligned}
& \lim _{n \rightarrow+\infty} \sum_{i, j=1}^{d} \int_{0}^{t} d \tau\left(\hat{v}_{n, x_{i}}(., \tau), k_{i, j}(., \tau) u_{V, \varphi, x_{j}}(., \tau)\right)_{2} \\
= & \sum_{i, j=1}^{d} \int_{0}^{t} d \tau\left(v_{x_{i}}(., \tau), k_{i, j}(., \tau) u_{V, \varphi, x_{j}}(., \tau)\right)_{2}
\end{aligned}
$$

and

$$
\lim _{n \rightarrow+\infty} \int_{0}^{t} d \tau\left(\hat{v}_{n}(., \tau), g\left(u_{V, \varphi}(., \tau)\right)\right)_{2}=\int_{0}^{t} d \tau\left(v(., \tau), g\left(u_{V, \varphi}(., \tau)\right)\right)_{2}
$$

$\mathbb{P}$-a.s. for every $t \in[0, T]$.

Proof. Regarding (23) we have

$$
\begin{aligned}
& \int_{0}^{t} d \tau\left|\left(v_{\tau}(., \tau)-\hat{v}_{n, \tau}(., \tau), u_{V, \varphi}(., \tau)\right)_{2}\right| \\
\leq & \left\|u_{V, \varphi}\right\|_{L^{2}\left(0, T ; H^{1}(D)\right)}\left(\int_{D \times(0, T)} d x d \tau\left|v_{\tau}(x, \tau)-\hat{v}_{n, \tau}(x, \tau)\right|^{2}\right)^{\frac{1}{2}} \\
\leq & \left\|u_{V, \varphi}\right\|_{L^{2}\left(0, T ; H^{1}(D)\right)}\left\|v-\hat{v}_{n}\right\|_{1,2, T} \rightarrow 0
\end{aligned}
$$

as $n \rightarrow+\infty \mathbb{P}$-a.s., by using successively Schwarz inequalities in $L^{2}(D)$ and on the time interval $(0, T)$ along with (11) and (22). The proof of (24) follows from similar arguments. Thus, noting that the matrix elements $k_{i, j}$ are uniformly bounded for all $i, j \in\{1, \ldots, d\}$ as a consequence of (3), we eventually get

$$
\begin{aligned}
& \sum_{i, j=1}^{d} \int_{0}^{t} d \tau\left|\left(v_{x_{i}}(., \tau)-\hat{v}_{n, x_{i}}(., \tau), k_{i, j}(., \tau) u_{V, \varphi, x_{j}}(., \tau)\right)_{2}\right| \\
\leq & c\left\|u_{V, \varphi}\right\|_{L^{2}\left(0, T ; H^{1}(D)\right)} \sum_{j=1}^{d}\left(\int_{D \times(0, T)} d x d \tau\left|v_{x_{j}}(x, \tau)-\hat{v}_{n, x_{j}}(x, \tau)\right|^{2}\right)^{\frac{1}{2}} \\
\leq & c\left\|u_{V, \varphi}\right\|_{L^{2}\left(0, T ; H^{1}(D)\right)}\left\|v-\hat{v}_{n}\right\|_{1,2, T} \rightarrow 0
\end{aligned}
$$

as $n \rightarrow+\infty \mathbb{P}$-a.s., where $c>0$ is a constant depending only on $\underline{k}, \bar{k}$ and $d$. In a similar way for (25) we have

$$
\begin{aligned}
& \lim _{n \rightarrow+\infty} \int_{0}^{t} d \tau\left|\left(v(., \tau)-\hat{v}_{n}(., \tau), g\left(u_{V, \varphi}(., \tau)\right)\right)_{2}\right| \\
\leq & \left(c_{1}+c_{2}\left\|u_{V, \varphi}\right\|_{L^{2}\left(0, T ; H^{1}(D)\right)}\right) \lim _{n \rightarrow+\infty}\left\|v-\hat{v}_{n}\right\|_{1,2, T}=0
\end{aligned}
$$

$\mathbb{P}$.-a.s. with $c_{1,2}>0$, which follows from the fact that $g$ is Lipschitz continuous and $|D|<+\infty$ where $|D|$ stands for the Lebesgue measure of $D$.

In the sequel we will also need approximation properties for the remaining terms in (12) that are similar to those of the above proposition. However, the 
proof of those properties and eventually of the main theorem will require two more preparatory results. The first one is:

Lemma 2. Let $\mathcal{H}(D \times(0, T))$ be the Hilbert space defined in Section 1 . Then there exists the continuous trace embedding

$$
\mathcal{H}(D \times(0, T)) \rightarrow L^{2}(D \times\{\tau=t\})
$$

valid for each $t \in[0, T]$ and every $d \in \mathbb{N}^{+}$. Moreover, there also exists the continuous embedding

$$
\mathcal{H}(D \times(0, T)) \rightarrow \mathcal{B}^{\alpha, 2}\left([0, T] ; L^{2}(D)\right)
$$

where $\mathcal{B}^{\alpha, 2}\left([0, T] ; L^{2}(D)\right)$ is endowed with norm [7).

Proof. It is sufficient to prove the result for $H^{1}(D \times(0, T))$. Let $v \in$ $H^{1}(D \times(0, T))$ and let us write momentarily $\|\cdot\|_{1,2, H^{1}((0, T))}$ for the norm in $H^{1}((0, T))$. From (9) and Fubini's theorem we infer that $x \mapsto v(x, t) \in L^{2}(D)$ for almost every $t$, and more importantly that $t \mapsto v(x, t) \in H^{1}((0, T))$ for almost every $x \in D$. Therefore, writing $\mathcal{C}([0, T])$ for the space of all continuous functions defined on $[0, T]$ endowed with the uniform norm $\|\cdot\|_{\infty, T}$ we have $t \mapsto v(x, t) \in \mathcal{C}([0, T])$ by virtue of the embedding $H^{1}((0, T)) \hookrightarrow \mathcal{C}([0, T])$. Consequently we obtain

$$
|v(x, t)| \leq\|v(x, .)\|_{\infty, T} \leq c\|v(x, .)\|_{1,2, H^{1}((0, T))}
$$

for each $t \in[0, T]$ and almost every $x \in D$ for some $c>0$, and thereby the desired result

$$
\int_{D} d x|v(x, t)|^{2} \leq c^{2} \int_{D} d x\|v(x, .)\|_{1,2, H^{1}((0, T))}^{2} \leq c^{2}\|v\|_{1,2, T}^{2}
$$

according to (9). As for the proof of the second embedding, we note that for every $v \in H^{1}(D \times(0, T))$ and $t \geq \tau$ we have

$$
\begin{aligned}
& \|v(., t)-v(., \tau)\|_{2} \\
\leq & (t-\tau)^{\frac{1}{2}}\left(\int_{D \times(0, T)} d x d \sigma\left|v_{\sigma}(x, \sigma)\right|^{2}\right)^{\frac{1}{2}} \leq(t-\tau)^{\frac{1}{2}}\|v\|_{1,2, T}
\end{aligned}
$$

by (9), and therefore

$$
\begin{gathered}
\|v\|_{\alpha, 2, T}^{2}=\left(\sup _{t \in[0, T]}\|v(., t)\|_{2}\right)^{2}+\int_{0}^{T} d t\left(\int_{0}^{t} d \tau \frac{\|v(., t)-v(., \tau)\|_{2}}{(t-\tau)^{\alpha+1}}\right)^{2} \\
\leq c\|v\|_{1,2, T}^{2}+\int_{0}^{T} d t\left(\int_{0}^{t} d \tau(t-\tau)^{-\alpha-\frac{1}{2}}\right)^{2}\|v\|_{1,2, T}^{2} \leq c\|v\|_{1,2, T}^{2}
\end{gathered}
$$


by virtue of (26) and the fact that $\alpha<\frac{1}{2}$, changing the value of $c$ whenever necessary.

The desired approximation property for the stochastic integral in (12) will now rest on (27) and on the following estimate, which also shows that (13) is Hölder continuous with respect to the time variable:

Lemma 3. Let us consider the stochastic integral as defined in (13). Then there exists a $\mathbb{P}$-a.s. finite, positive random variable $r_{\alpha}^{\mathrm{H}}$ such that the estimate

$$
\begin{aligned}
& \left|\int_{t^{*}}^{t} \int_{D} d x v(x, \tau) h\left(u_{V, \varphi}(x, \tau)\right) W^{\mathrm{H}}(x, d \tau)\right| \\
\leq & r_{\alpha}^{\mathrm{H}}\left(1+\left\|u_{V, \varphi}\right\|_{\alpha, 2, T}\right)\|v\|_{\alpha, 2, T}\left|t^{*}-t\right|^{\frac{1}{2}}
\end{aligned}
$$

holds $\mathbb{P}$-a.s. for every $v \in \mathcal{B}^{\alpha, 2}\left([0, T] ; L^{2}(D)\right)$ and all $t, t^{*} \in[0, T]$.

We defer the proof of this lemma to the Appendix, as it requires technical tools regarding generalized Stieltjes integrals that do not directly pertain to the main core of this article.

The expected approximation property of the stochastic integral is then the following:

Proposition 3. Let $v \in \mathcal{H}(D \times(0, T))$ and $\left(\hat{v}_{n}\right)_{n \in \mathbb{N}^{+}}$be as in Proposition 2. Then we have

$$
\begin{aligned}
& \lim _{n \rightarrow+\infty} \int_{0}^{t} \int_{D} d x \hat{v}_{n}(x, \tau) h\left(u_{V, \varphi}(x, \tau)\right) W^{\mathrm{H}}(x, d \tau) \\
= & \int_{0}^{t} \int_{D} d x v(x, \tau) h\left(u_{V, \varphi}(x, \tau)\right) W^{\mathrm{H}}(x, d \tau)
\end{aligned}
$$

$\mathbb{P}$-a.s. for every $t \in[0, T]$.

Proof. From Relation (28) and embedding (27) we have

$$
\begin{aligned}
& \left|\int_{0}^{t} \int_{D} d x\left(v(x, \tau)-\hat{v}_{n}(x, \tau)\right) h\left(u_{V, \varphi}(x, \tau)\right) W^{\mathrm{H}}(x, d \tau)\right| \\
\leq & c_{\alpha}^{\mathrm{H}}\left(1+\left\|u_{V, \varphi}\right\|_{\alpha, 2, T}\right)\left\|v-\hat{v}_{n}\right\|_{1,2, T}
\end{aligned}
$$

$\mathbb{P}$-a.s. for some $c>0$, hence the desired result from (22).

The preceding considerations now lead to the following: 
Proof of the main result. Let $v \in \mathcal{H}(D \times(0, T))$ and let $\left(\hat{v}_{n}\right)_{n \in \mathbb{N}^{+}}$be as in (22). According to Lemma 1 we have

$$
\begin{aligned}
\left(\hat{v}_{n}(., t), u_{V, \varphi}(., t)\right)_{2}= & \left(\hat{v}_{n}(., 0), \varphi\right)_{2}+\int_{0}^{t} d \tau\left(\hat{v}_{n, \tau}(., \tau), u_{V, \varphi}(., \tau)\right)_{2} \\
& -\sum_{i, j=1}^{d} \int_{0}^{t} d \tau\left(\hat{v}_{n, x_{i}}(., \tau), k_{i, j}(., \tau) u_{V, \varphi, x_{j}}(., \tau)\right)_{2} \\
& +\int_{0}^{t} d \tau\left(\hat{v}_{n}(., \tau), g\left(u_{V, \varphi}(., \tau)\right)\right)_{2} \\
& +\int_{0}^{t} \int_{D} d x \hat{v}_{n}(x, \tau) h\left(u_{V, \varphi}(x, \tau)\right) W^{\mathrm{H}}(x, d \tau)
\end{aligned}
$$

$\mathbb{P}$-a.s. for each $t \in[0, T]$ and every $n$, and we already know that each integral on the right-hand side of this expression converges to the desired integral in (12) according to Propositions 2 and 3. Let us now investigate the term on the left-hand side. From Schwarz inequality in $L^{2}(D)$ and (7) we have

$$
\begin{aligned}
& \left|\left(v(., t)-\hat{v}_{n}(., t), u_{V, \varphi}(., t)\right)_{2}\right| \\
\leq & \left\|u_{V, \varphi}\right\|_{\alpha, 2, T} \sup _{t \in[0, T]}\left\|v(., t)-\hat{v}_{n}(., t)\right\|_{2} \\
\leq & \left\|u_{V, \varphi}\right\|_{\alpha, 2, T}\left\|v-\hat{v}_{n}\right\|_{\alpha, 2, T} \leq c\left\|u_{V, \varphi}\right\|_{\alpha, 2, T}\left\|v-\hat{v}_{n}\right\|_{1,2, T} \rightarrow 0
\end{aligned}
$$

$\mathbb{P}$-a.s. as $n \rightarrow+\infty$ for some $c>0$ by virtue of (22) and (27). Therefore we get

$$
\lim _{n \rightarrow+\infty}\left(\hat{v}_{n}(., t), u_{V, \varphi}(., t)\right)_{2}=\left(v(., t), u_{V, \varphi}(., t)\right)_{2}
$$

$\mathbb{P}$-a.s. uniformly in $t \in[0, T]$, where $v(., t)$ stands for the trace of $v$ in the sense of (26). Passing to the limit in (29) we then obtain for the remaining term

$$
\begin{aligned}
\lim _{n \rightarrow+\infty}\left(\hat{v}_{n}(., 0), \varphi\right)_{2}= & \left(v(., t), u_{V, \varphi}(., t)\right)_{2}-\int_{0}^{t} d \tau\left(v_{\tau}(., \tau), u_{V, \varphi}(., \tau)\right)_{2} \\
& +\sum_{i, j=1}^{d} \int_{0}^{t} d \tau\left(v_{x_{i}}(., \tau), k_{i, j}(., \tau) u_{V, \varphi, x_{j}}(., \tau)\right)_{2} \\
& -\int_{0}^{t} d \tau\left(v(., \tau), g\left(u_{V, \varphi}(., \tau)\right)\right)_{2} \\
& -\int_{0}^{t} \int_{D} d x v(x, \tau) h\left(u_{V, \varphi}(x, \tau)\right) W^{\mathrm{H}}(x, d \tau)
\end{aligned}
$$

$\mathbb{P}$-a.s. for every $t \in[0, T]$, hence

$$
\lim _{n \rightarrow+\infty}\left(\hat{v}_{n}(., 0), \varphi\right)_{2}=\left(v(., 0), u_{V, \varphi}(., 0)\right)_{2}
$$

by choosing $t=0$. But from (16) at $t=0$ we have $\left(v, u_{V, \varphi}(., 0)-\varphi\right)_{2}=0$ for every $v \in H^{1}(D)$ and thereby for every smooth and compactly supported 
$v \in \mathcal{C}_{c}^{1}(D)$, the latter space being dense in $L^{2}(D)$. Therefore $u_{V, \varphi}(., 0)-\varphi$ is orthogonal to $L^{2}(D)$, hence $u_{V, \varphi}(., 0)=\varphi$ so that

$$
\lim _{n \rightarrow+\infty}\left(\hat{v}_{n}(., 0), \varphi\right)_{2}=(v(., 0), \varphi)_{2} .
$$

The substitution of the preceding expression into (30) then proves (12) for every $v \in \mathcal{H}(D \times(0, T))$. Finally, the proof that $u_{V, \varphi}$ is the unique variational solution to (2) satisfying (16) when $h$ is an affine function is identical to that carried out in [17]. Therefore, let $\tilde{u}_{V, \varphi}$ be another variational solution to (2) satisfying (12). We then have $\tilde{u}_{V, \varphi}=u_{V, \varphi} \mathbb{P}$-a.s. as a solution to (16), hence also as a solution to (12).

REMARKs. (1) The preceding considerations show that there are actually two distinct types of equivalent variational solutions to (2), to wit, one that satisfies (16) which involves test functions independent of time, and one satisfying (12). As was done in [17] where the class of problems given by (2) was defined and analysed on a bounded domain $D$ satisfying the cone condition, we may refer to them as variational solutions of type I and II, respectively. Moreover, for the kind of equations considered in this article and to the best of our knowledge, the problem of uniqueness of the solution in case of a general nonlinearity $h$ in the noise term remains open.

(2) If $D$ is a bounded domain that satisfies the cone condition, the natural space of test functions for variational solutions of type II is $H^{1}(D \times(0, T))$, as was shown in [17 by first using (16) to prove (12) for polynomial test functions, then for smooth test functions by invoking the $\mathcal{C}^{1}$-version of Weierstrass' approximation theorem, and eventually for functions in $H^{1}(D \times(0, T))$ by a density argument. It is not possible to argue in that way when $D$ is unbounded and merely satisfies Hypothesis (D). Thus, it is still true that the space $\mathcal{C}^{1}(\bar{D} \times[0, T])$ of all uniformly continuous and uniformly bounded functions on $D \times(0, T)$ is dense in $H^{1}(D \times(0, T))$ if we assume in addition that $D$ and therefore $D \times(0, T)$ satisfy the so-called segment condition (see, e.g. 1]). However, we can no longer approximate functions in $\mathcal{C}^{1}(\bar{D} \times[0, T])$ by polynomials in a suitable topology to make such approximations worthwile in the context of this article (an approximation of the functions in $\mathcal{C}^{1}(\bar{D} \times[0, T])$ by polynomials is still possible if one endows $\mathcal{C}^{1}(D \times(0, T))$ with the structure of a Fréchet space, see, e.g., Section 3 of Chapter 3 in 12, but this did not turn out to be strong enough in our case). We therefore bypassed the difficulty by constructing the space $\mathcal{H}(D \times(0, T))$ in Section 1, but the determination of the relative size of this space within $H^{1}(D \times(0, T))$ remains an open problem at the present time.

We complete this section by illustrating the above results by means of two examples. We begin with:

Proposition 4. Let us consider the two-dimensional domain

$$
D=\left\{\left(x_{1}, x_{2}\right) \in \mathbb{R}^{2}: x_{1}>0, \quad 0<x_{2}<b\left(x_{1}\right)\right\}
$$


where the positive boundary curve $b \in \mathcal{C}^{1}\left(\mathbb{R}_{0}^{+}\right)$is decreasing, has a bounded derivative and satisfies

$$
\lim _{s \rightarrow+\infty} \frac{b(s+\epsilon)}{b(s)}=0
$$

for every $\epsilon>0$. Then the conditions of Hypothesis (D) in Section 1 hold.

Proof. For every $N \in \mathbb{N}^{+}$let us define the bounded open set

$$
D_{N}:=\left\{\left(x_{1}, x_{2}\right) \in \mathbb{R}^{2}: 0<x_{1}<N\right\} \cap D .
$$

Then we have $D_{N} \subset D_{N+1} \subset D$ and $D_{N}$ satisfies the cone condition, so that compact embedding (14) holds by the Rellich-Kondrachov theorem. Furthermore, the properties of the flow associated with $D$ in the sense of Example 6.46 and Theorem 6.47 in 1 by means of the function $b$ satisfying (32) imply that for every $u \in H^{1}(D)$, the inequality

$$
\int_{D \backslash D_{N}} d x|u(x)|^{2} \leq c_{N}\|u\|_{1,2}^{2}
$$

is valid with $\lim _{N \rightarrow+\infty} c_{N}=0$. Consequently, for each $\epsilon>0$ there exists $N_{\epsilon} \in \mathbb{N}^{+}$such that (15) holds for each $u$ belonging to a bounded set of $H^{1}(D)$.

We conclude that for a domain of the form (31) the embedding $H^{1}(D) \hookrightarrow$ $L^{2}(D)$ is compact, so that our main theorem holds true in this case. Typical examples of boundary functions satisfying the above conditions include $b(x)=$ $\exp \left[-x^{1+\delta}\right]$ where $\delta>0$, whereas the mere exponential $b(x)=\exp [-x]$ does not belong to that class of curves. In fact, since $b$ is decreasing it follows easily from (32) that

$$
\lim _{s \rightarrow+\infty} \exp [s] b(s)=0 .
$$

Generally speaking, it is in fact the finiteness of the Lebesgue measure of $D$, along with the rapid decrease of the measure of the part of $D$ situated outside the disk of radius $R$ centered at the origin as $R \rightarrow+\infty$, which makes all this possible.

Our second example refers to the horn-shaped region discussed in 2, for which our main theorem is also valid:

Proposition 5. Let us consider the three-dimensional domain

$$
D=\left\{\left(x_{1}, x_{2}, x_{3}\right) \in \mathbb{R}^{3}: x_{3}>0, \quad 0<x_{1}^{2}+x_{2}^{2}<b^{2}\left(x_{3}\right)\right\}
$$

where the boundary curve $b$ satisfies the same hypotheses as in Proposition 4. Then the conditions of Hypothesis (D) in Section 1 hold. 
Proof. The argument is similar to that given in the proof of Proposition 4 if we define

$$
D_{N}:=\left\{\left(x_{1}, x_{2}, x_{3}\right) \in \mathbb{R}^{3}: 0<x_{3}<N\right\} \cap D
$$

for every $N \in \mathbb{N}^{+}$. Thus the compactness of embedding (14) along with (15) hold.

Acknowledgements The research of M. D. was supported in part by the FCT of the Portuguese government under grant FCT UID/MAT/00208/2013. He would like to thank Professors A. B. Cruzeiro, P.-A. Vuillermot and J. C. Zambrini for their very kind invitation to the GFMUL, where this work was completed. R. T. would like to extend her thanks and express her appreciation and gratitude to the entire staff of the IECL for their warm hospitality and strong support during the completion of her Ph.D. dissertation there. The research of P.-A. V. was supported in part by the FCT of the Portuguese government under grant PDTC/MAT-STA/0975/2014.

Appendix: some remarks on generalized Stieltjes integrals and proof of Lemma 3

The following considerations constitute a necessary modification of the theory developed in [16, needed to take into account the fact that we are dealing here with an infinite sequence of Hurst parameters. For every $i \in \mathbb{N}^{+}$let us introduce the random variable

$$
\Lambda_{\alpha}^{\mathrm{H}_{i}}=\frac{\sin (\pi \alpha)}{\pi} \sup _{0 \leq t<t^{*} \leq T}\left|\frac{B^{\mathrm{H}_{i}}(t)-B^{\mathrm{H}_{i}}\left(t^{*}\right)}{\left(t^{*}-t\right)^{1-\alpha}}+(1-\alpha) \int_{t}^{t^{*}} d \tau \frac{B^{\mathrm{H}_{i}}(t)-B^{\mathrm{H}_{i}}(\tau)}{(\tau-t)^{2-\alpha}}\right|
$$

where $\alpha$ is the fixed parameter chosen in Section 1, for which we have the inequality

$$
\Lambda_{\alpha}^{\mathrm{H}_{i}} \leq \sup _{0 \leq t<t^{*} \leq T}\left(\frac{\left|B^{\mathrm{H}_{i}}\left(t^{*}\right)-B^{\mathrm{H}_{i}}(t)\right|}{\left(t^{*}-t\right)^{1-\alpha}}+(1-\alpha) \int_{t}^{t^{*}} d \tau \frac{\left|B^{\mathrm{H}_{i}}(\tau)-B^{\mathrm{H}_{i}}(t)\right|}{(\tau-t)^{2-\alpha}}\right) .
$$

For reasons that will soon be apparent, we first need to get appropriate estimates for the moments of (33) that are uniform in $i \in \mathbb{N}^{+}$, and to achieve this suitable upper bounds for the increments of $B^{\mathrm{H}}(t)$. Indeed the main result that will lead to the proof of Lemma 3 is:

Proposition A. 1. Let $\left(B^{\mathrm{H}_{i}}(t)\right)_{i \in \mathbb{N}^{+}}$be the one-dimensional, independent fractional Brownian motions introduced in Section 1, where the sequence $\mathbf{H}=$ $\left(\mathrm{H}_{i}\right)_{i \in \mathbb{N}^{+}}$satisfies (4). Then for each $i \in \mathbb{N}^{+}$we have

$$
\sup _{i \in \mathbb{N}^{+}} \mathbb{E}\left|\Lambda_{\alpha}^{\mathrm{H}_{i}}\right|^{p}<+\infty
$$

for every $p \in[1,+\infty)$. 
The proof of this proposition rests on the following particular case of the Garsia-Rodemich-Rumsey inequality, obtained by rescaling the basic estimate stated in Lemma 1.1 of $[8]$ to establish its validity on $[0, T]$ rather than just on $[0,1]$, and by applying the particular choice of the functions involved made at the very beginning of [10]:

Lemma A. 1. Let $f:[0, T] \mapsto \mathbb{R}$ be continuous, let $q \in[1,+\infty)$ and $\beta \in\left(\frac{1}{q},+\infty\right)$. If the integral between parentheses in (36) is finite, then the inequality

$$
\left|f\left(t^{*}\right)-f(t)\right| \leq c_{\beta, q, T}\left(\int_{0}^{T} \int_{0}^{T} d \sigma d \tau \frac{|f(\sigma)-f(\tau)|^{q}}{|\sigma-\tau|^{\beta q+1}}\right)^{\frac{1}{q}}\left|t^{*}-t\right|^{\beta-\frac{1}{q}}
$$

holds for all $t, t^{*} \in[0, T]$, where

$$
c_{\beta, q, T}=c_{T}\left(\beta+\frac{1}{q}\right)\left(\beta-\frac{1}{q}\right)^{-1}
$$

and where $c_{T}>0$ depends only on $T$.

The upper bounds for the increments of $B^{\mathrm{H}_{i}}(t)$ we need turn out to be provided by (36) for particular values of the parameters. In all that follows we write $\epsilon$ for a fixed auxiliary quantity which we can eventually express in terms of the constants $\alpha, \gamma$ and $\underline{\mathrm{H}}$ :

Lemma A. 2. Let us fix $\epsilon \in\left(0, \frac{1}{\gamma+1}\right)$ where $\gamma \in(0,1]$ is the constant of Hypothesis (L). Then for every $i \in \mathbb{N}^{+}$there exists a positive, $\mathbb{P}$-a.s. finite random variable $\Theta_{i, \epsilon, T}$ such that the inequality

$$
\left|B^{\mathrm{H}_{i}}\left(t^{*}\right)-B^{\mathrm{H}_{i}}(t)\right| \leq c_{\gamma, \epsilon, T} \Theta_{i, \epsilon, T}\left|t^{*}-t\right|^{\underline{\mathrm{H}}-\epsilon}
$$

holds $\mathbb{P}$-a.s. for all $t, t^{*} \in[0, T]$, where $c_{\gamma, \epsilon, T}>0$ depends only on $\gamma, \epsilon$ and $T$. Moreover we have

$$
\sup _{i \in \mathbb{N}^{+}} \mathbb{E}\left|\Theta_{i, \epsilon, T}\right|^{p}<+\infty
$$

for every $p \in[1,+\infty)$.

Proof. Let us first define the random variable

$$
\tilde{\Theta}_{i, \epsilon, T}:=\int_{0}^{T} \int_{0}^{T} d \sigma d \tau \frac{\left|B^{\mathrm{H}_{i}}(\sigma)-B^{\mathrm{H}_{i}}(\tau)\right|^{\frac{2}{\epsilon}}}{|\sigma-\tau|^{\frac{2 \mathrm{H}_{i}}{\epsilon}}}
$$

and prove that

$$
\sup _{i \in \mathbb{N}^{+}} \mathbb{E}\left|\tilde{\Theta}_{i, \epsilon, T}\right|^{p}<+\infty
$$


for every $p \in[1,+\infty)$. For any $r \in\left[\frac{2}{\epsilon},+\infty\right)$ we have $p:=\frac{r \epsilon}{2} \in[1,+\infty)$ so that on the one hand we obtain

$$
\begin{aligned}
\mathbb{E}\left|\tilde{\Theta}_{i, \epsilon, T}\right|^{p} & =\mathbb{E}\left(\int_{0}^{T} \int_{0}^{T} d \sigma d \tau \frac{\left|B^{\mathrm{H}_{i}}(\sigma)-B^{\mathrm{H}_{i}}(\tau)\right|^{\frac{2}{\epsilon}}}{|\sigma-\tau|^{\frac{2 \mathrm{H}_{i}}{\epsilon}}}\right)^{\frac{r \epsilon}{2}} \\
& \leq\left(\int_{0}^{T} \int_{0}^{T} d \sigma d \tau\left(\mathbb{E} \frac{\left|B^{\mathrm{H}_{i}}(\sigma)-B^{\mathrm{H}_{i}}(\tau)\right|^{r}}{|\sigma-\tau|^{r \mathrm{H}_{i}}}\right)^{\frac{2}{r \epsilon}}\right)^{\frac{r \epsilon}{2}}
\end{aligned}
$$

from Minkowski's integral inequality. On the other hand, from the basic properties of the one-dimensional fractional Brownian motion we have

$$
\mathbb{E}\left|B^{\mathrm{H}_{i}}(\sigma)-B^{\mathrm{H}_{i}}(\tau)\right|^{r} \leq c_{r}|\sigma-\tau|^{r \mathrm{H}_{i}}
$$

for some constant $c_{r}$ depending only on $r$, so that the substitution of this expression into (41) annihilates the dependence in $\mathrm{H}_{i}$, thus leading to

$$
\mathbb{E}\left|\tilde{\Theta}_{i, \epsilon, T}\right|^{p} \leq c_{r} T^{2 p}<+\infty
$$

uniformly in $i$, which is (40). Let us now choose $q=\frac{2}{\epsilon}$ and $\beta_{i}=\mathrm{H}_{i}-\frac{\epsilon}{2}$ for every $i \in \mathbb{N}^{+}$in Lemma A. 1 . Then clearly $q \in[1,+\infty)$ and $\beta_{i} \in\left(\frac{\epsilon}{2},+\infty\right)$ by virtue of (40), and since (40) implies in particular that $\tilde{\Theta}_{i, \epsilon, T}<+\infty \mathbb{P}$-a.s. we may thus apply (36) to obtain

$$
\begin{aligned}
& \left|B^{\mathrm{H}_{i}}\left(t^{*}\right)-B^{\mathrm{H}_{i}}(t)\right| \\
\leq & c_{\beta_{i}, q, T}\left(\int_{0}^{T} \int_{0}^{T} d \sigma d \tau \frac{\left|B^{\mathrm{H}_{i}}(\sigma)-B^{\mathrm{H}_{i}}(\tau)\right|^{\frac{2}{\epsilon}}}{|\sigma-\tau|^{\frac{2 \mathrm{H}_{i}}{\epsilon}}}\right)^{\frac{\epsilon}{2}}\left|t^{*}-t\right|^{\mathrm{H}_{i}-\epsilon} \\
\leq & \tilde{c}_{\beta_{i}, q, T}\left(\int_{0}^{T} \int_{0}^{T} d \sigma d \tau \frac{\left|B^{\mathrm{H}_{i}}(\sigma)-B^{\mathrm{H}_{i}}(\tau)\right|^{\frac{2}{\epsilon}}}{|\sigma-\tau|^{\frac{2 \mathrm{H}_{i}}{\epsilon}}}\right)^{\frac{\epsilon}{2}}\left|t^{*}-t\right|^{\underline{\underline{H}}-\epsilon} \\
= & \tilde{c}_{\beta_{i}, q, T} \tilde{\Theta}_{i, \epsilon, T}^{\frac{\epsilon}{2}}\left|t^{*}-t\right|^{\underline{\underline{H}}-\epsilon}
\end{aligned}
$$

$\mathbb{P}$-a.s. for all $t, t^{*} \in[0, T]$, where $\tilde{c}_{\beta_{i}, q, T}$ differs from $c_{\beta_{i}, q, T}$ by a trivial factor depending only on $T$ and $\underline{\mathrm{H}}$. In order to get (38) it is thus sufficient to take

$$
\Theta_{i, \epsilon, T}:=\tilde{\Theta}_{i, \epsilon, T}^{\frac{\epsilon}{2}}
$$

and prove that

$$
\sup _{i \in \mathbb{N}^{+}} \tilde{c}_{\beta_{i}, q, T}<+\infty
$$

along with (39). Ignoring the trivial dependence in $T$ and $\underline{\mathrm{H}}$ in $\tilde{c}_{\beta_{i}, q, T}$ we first infer from (37) that the simple estimate

$$
\left(\beta_{i}+\frac{\epsilon}{2}\right)\left(\beta_{i}-\frac{\epsilon}{2}\right)^{-1}=\mathrm{H}_{i}\left(\mathrm{H}_{i}-\epsilon\right)^{-1} \leq\left(\frac{1}{\gamma+1}-\epsilon\right)^{-1}<+\infty
$$


holds uniformly in $i$ thanks to (4) and our choice of $\epsilon$, which gives (43). Finally, let us partition the probability space as

$$
\Omega=\left\{\omega \in \Omega: \tilde{\Theta}_{i, \epsilon, T}(\omega) \leq 1\right\} \cup\left\{\omega \in \Omega: \tilde{\Theta}_{i, \epsilon, T}(\omega)>1\right\}
$$

and split the expectation functional accordingly. From (42) it is then plain that

$$
\sup _{i \in \mathbb{N}^{+}} \mathbb{E}\left|\Theta_{i, \epsilon, T}\right|^{p}=\sup _{i \in \mathbb{N}^{+}} \mathbb{E}\left|\tilde{\Theta}_{i, \epsilon, T}\right|^{\frac{p \epsilon}{2}} \leq 1+\sup _{i \in \mathbb{N}^{+}} \mathbb{E}\left|\tilde{\Theta}_{i, \epsilon, T}\right|^{p}<+\infty
$$

according to (40) since $\frac{\epsilon}{2} \leq 1$, which is the desired result.

The proof of (35) then follows from (39) and from the substitution of (38) into (34) provided we impose a further restriction on the parameter $\epsilon$ to make the singularities integrable:

Proof of Proposition A. 1. We first notice that

$$
\underline{\mathrm{H}}-1+\alpha<\frac{1}{\gamma+1}
$$

as a consequence of the conditions we imposed on these parameters in Section 1. Then, fixing $\epsilon \in(0, \underline{\mathrm{H}}-1+\alpha)$ we may substitute (38) into (34) to obtain

$$
\Lambda_{\alpha}^{\mathrm{H}_{i}} \leq c_{\gamma, \epsilon, T} T^{\mathrm{H}-\epsilon-1+\alpha} \frac{\underline{\mathrm{H}}-\epsilon+\alpha}{\underline{\mathrm{H}}-\epsilon-1+\alpha} \Theta_{i, \epsilon, T}<+\infty
$$

$\mathbb{P}$-a.s. after an explicit integration, where the prefactor is uniform in $i \in \mathbb{N}^{+}$. Therefore, (35) indeed follows from (39).

REMARK. To follow up on our remark preceding the statement of Lemma A. 2., we see a posteriori that we could have chosen for instance $\epsilon=\frac{1}{2}(\underline{\mathrm{H}}-1+\alpha)$ throughout.

We are now ready for the following:

Proof of Lemma 3. We begin by estimating each integral on the righthand side of (13). For every $i \in \mathbb{N}^{+}$let us set

$$
f_{i}(\tau):=\left(v(., \tau), h\left(u_{V, \varphi}(., \tau)\right) e_{i}\right)_{2} .
$$

Then, from the basic estimate (4.11) in Proposition 4.1 of [16] regarding generalized Stieltjes integrals we infer that the inequality

$$
\left|\int_{t^{*}}^{t} f_{i}(\tau) B^{\mathrm{H}_{i}}(d \tau)\right| \leq \Lambda_{\alpha}^{\mathrm{H}_{i}} \int_{t^{*}}^{t} d \tau\left(\frac{\left|f_{i}(\tau)\right|}{\left(\tau-t^{*}\right)^{\alpha}}+\alpha \int_{t^{*}}^{\tau} d \rho \frac{\left|f_{i}(\tau)-f_{i}(\rho)\right|}{(\tau-\rho)^{\alpha+1}}\right)
$$


holds $\mathbb{P}$-a.s., where $\Lambda_{\alpha}^{\mathrm{H}_{i}}$ is given by (33). Furthermore, from Schwarz inequality and the basic hypotheses of Section 1 we have

$$
\begin{aligned}
\left|f_{i}(\tau)\right| & \leq c\left\|e_{i}\right\|_{\infty}\left(1+\left\|u_{V, \varphi}(., \tau)\right\|_{2}\right)\|v(., \tau)\|_{2} \\
& \leq c\left\|e_{i}\right\|_{\infty}\left(1+\left\|u_{V, \varphi}\right\|_{\alpha, 2, T}\right)\|v\|_{\alpha, 2, T}
\end{aligned}
$$

where the second inequality follows from (7), and similarly

$$
\begin{aligned}
& \left|f_{i}(\tau)-f_{i}(\rho)\right| \\
\leq & c\left\|e_{i}\right\|_{\infty}\left\|u_{V, \varphi}(., \tau)-u_{V, \varphi}(., \rho)\right\|_{2}\|v\|_{\alpha, 2, T} \\
& +c\left\|e_{i}\right\|_{\infty}\left(1+\left\|u_{V, \varphi}\right\|_{\alpha, 2, T}\right)\|v(., \tau)-v(., \rho)\|_{2}
\end{aligned}
$$

for all $\rho, \tau \in[0, T]$, for some constant $c>0$. Consequently, on the one hand the substitution of (45) into the first integral on the right-hand side of (44) gives

$$
\int_{t^{*}}^{t} d \tau \frac{\left|f_{i}(\tau)\right|}{\left(\tau-t^{*}\right)^{\alpha}} \leq c\left\|e_{i}\right\|_{\infty}\left(1+\left\|u_{V, \varphi}\right\|_{\alpha, 2, T}\right)\|v\|_{\alpha, 2, T}\left|t^{*}-t\right|^{1-\alpha}
$$

by direct integration. On the other hand, the substitution of (46) into the second integral on the right-hand side of (44), Schwarz inequality relative to the measure $d \tau$ and (77) lead to

$$
\int_{t^{*}}^{t} d \tau \int_{t^{*}}^{\tau} d \rho \frac{\left|f_{i}(\tau)-f_{i}(\rho)\right|}{(\tau-\rho)^{\alpha+1}} \leq c\left\|e_{i}\right\|_{\infty}\left(1+\left\|u_{V, \varphi}\right\|_{\alpha, 2, T}\right)\|v\|_{\alpha, 2, T}\left|t^{*}-t\right|^{\frac{1}{2}} .
$$

Therefore, with (47) and (48) into (44) we obtain

$$
\left|\int_{t^{*}}^{t} f_{i}(\tau) B^{\mathrm{H}_{i}}(d \tau)\right| \leq c\left\|e_{i}\right\|_{\infty} \Lambda_{\alpha}^{\mathrm{H}_{i}}\left(1+\left\|u_{V, \varphi}\right\|_{\alpha, 2, T}\right)\|v\|_{\alpha, 2, T}\left|t^{*}-t\right|^{\frac{1}{2}}
$$

for every $i \in \mathbb{N}^{+}$since $1-\alpha>\frac{1}{2}$ according to our original choice of this parameter. In order to prove (28) it is therefore sufficient to show that

$$
\sum_{i=1}^{+\infty} \sqrt{\lambda_{i}}\left\|e_{i}\right\|_{\infty} \Lambda_{\alpha}^{\mathrm{H}_{i}}<+\infty
$$

$\mathbb{P}$-a.s., for then

$$
\begin{aligned}
& \left|\int_{t^{*}}^{t} \int_{D} d x v(x, \tau) h\left(u_{V, \varphi}(x, \tau)\right) W^{\mathrm{H}}(x, d \tau)\right| \\
\leq & \left(c \sum_{i=1}^{+\infty} \sqrt{\lambda_{i}}\left\|e_{i}\right\|_{\infty} \Lambda_{\alpha}^{\mathrm{H}_{i}}\right)\left(1+\left\|u_{V, \varphi}\right\|_{\alpha, 2, T}\right)\|v\|_{\alpha, 2, T}\left|t^{*}-t\right|^{\frac{1}{2}}
\end{aligned}
$$

according to (13) and (49), with the obvious choice for $r_{\alpha}^{\mathrm{H}}$. But (50) follows from Relation (35) of Proposition A.1. with $p=1$ since spectral condition (6) holds. 


\section{References}

[1] Adams, R. A., Sobolev Spaces, Pure and Applied Mathematics Series 65, Academic Press, New York (1975).

[2] Adams, R. A., Fournier, J. J. F., Some imbedding theorems for Sobolev spaces, Canadian Journal of Mathematics 23 (1971) 517-530.

[3] Alvarez, A., López-Mimbela, J. A., Privault, N., Blowup estimates for a family of semilinear SPDE's with time-dependent coefficients, Differential Equations \& Applications 7 (2015) 201-219.

[4] Balan, R. M., Tudor, C. A., Stochastic heat equation with multiplicative fractional-colored noise, Journal of Theoretical Probability 23 (2010) 834870.

[5] Dozzi, M., Kolkovska E. T., López-Mimbela, J. A., Exponential functionals of Brownian motion and explosion times of a system of semilinear SPDEs, Stochastic Analysis and Applications 31 (2013) 975-991.

[6] Dozzi, M., Kolkovska E. T., López-Mimbela, J. A., Finite-time blowup and existence of global positive solutions of a semi-linear SPDE with fractional noise, in: Modern Stochastics and Applications, Springer Optimization and its Applications Series 90, Springer, New York, (2014) 95-108.

[7] Dozzi, M., López-Mimbela J. A., Finite-time blowup and existence of global positive solutions of a semi-linear SPDE, Stochastic Processes and their Applications 120 (2010) 767-776.

[8] Garsia, A., Rodemich, E., Rumsey, H., A real variable lemma and the continuity of paths of some Gaussian processes, Indiana University Mathematics Journal 20 (1971) 565-578.

[9] Guerra, J., Nualart, D., Stochastic differential equations driven by fractional Brownian motion and standard Brownian motion, Stochastic Analysis and Applications 26 (2008) 1053-1075.

[10] Hu, Y., LE, K., A multiparameter Garsia-Rodemich-Rumsey inequality and some applications, Stochastic Processes and their Applications 123 (2013) 3359-3377.

[11] Lv, G., DuAn, J., Impacts of noise on a class of partial differential equations, Journal of Differential Equations 258 (2015) 2196-2220.

[12] Kirillov, A. A., Gvishiani, A. D., Theorems and Problems in Functional Analysis, Problem Books in Mathematics 9, Springer, New York (1982). 
[13] Mishura, Y. S., Shevchenko, G. M., Existence and uniqueness of the solution of stochastic differential equation involving Wiener process and fractional Brownian motion with Hurst index $H>\frac{1}{2}$, Communications in Statistics-Theory and Methods 40 (2011) 3492-3508.

[14] Mishura, Y. S., Shevchenko, G. M., Mixed stochastic differential equations with long-range dependence: existence, uniqueness and convergence of solutions, Computer and Mathematics with Applications 64 (2012) 32173227 .

[15] Mishura, Y. S., Ralchenko, K., Shevchenko, G. M., Existence and uniqueness of mild solution to stochastic heat equation with white and fractional noises, Theory of Probability and Mathematical Statistics 98 (2018), in press.

[16] Nualart, D., RăşCAnu, A., Differential equations driven by fractional Brownian motion, Collect. Math. 53 (2002) 55-81.

[17] Nualart, D., Vuillermot, P. A., Variational solutions for partial differential equations driven by a fractional noise, Journal of Functional Analysis 232 (2006) 390-454.

[18] Stein, E., Singular Integrals and Differentiability Properties of Functions, Princeton University Press, Princeton, (1970).

[19] Vuillermot, P. A., On the time evolution of Bernstein processes associated with a class of parabolic equations, Discrete and Continuous Dynamical Systems Series B 23 (2018) 1073-1090. 